\title{
Epidemiological Characteristics of Gallbladder Cancer in Jeju Island: A Single-Center, Clinically Based, Age-Sex-Matched, Case-Control Study
}

\author{
Byung Hyo Cha
}

\begin{abstract}
Background: Gallbladder cancer (GBC) is a rare but highly invasive malignancy characterized by poor survival. In a national cancer survey, the age-standardized incidence rate of GBC was highest in Jeju Island among the 15 provinces in South Korea. The aim of this descriptive epidemiological study was to suggest the modifiable risk factors for this rare malignant disease in Jeju Island by performing an age-sex-matched casecontrol study. Materials and Methods: The case group included patients diagnosed with GBC at the Department of Internal Medicine of Cheju Halla General Hospital, Jeju, South Korea, within the 5-year study period. The control group consisted of age-sex-matched subjects selected from among the participants of the health promotion center at the same institute and in the same period. We compared 78 case-control pairs in terms of clinical variables such as histories of hypertension, diabetes, vascular occlusive disorders, alcohol and smoking consumption, obesity, and combined polypoid lesions of the gallbladder (PLG) or gallstone diseases (GSDs). Results: Among the relevant risk factors, alcohol consumption, parity $\geq 2$, PLG, and GSDs were significant risk factors in the univariate analysis. PLG $(p<0.01 ;$ OR, 51.1; 95\% confidence interval $[C I], 2.98-875.3)$ and GSD $(p<0.01$; OR, 54.9; 95\% CI, 3.00-1001.8) were associated risk factors of GBC in the multivariate analysis with the conditional logistic regression model. However, we failed to find any correlation between obesity and GBC. We also found a negative correlation between alcohol consumption history and GBC in the multivariate analysis (p < 0.01; OR, 0.06; 95\% CI, 0.01-0.31). Conclusions: These results suggest that combined PLG and GSDs are strongly associated with the GBC in Jeju Island and mild to moderate alcohol consumption may negatively correlate with GBC risk.
\end{abstract}

Keywords: Gallbladder cancer - risk factor - Gallstone disease - alcohol consumption

Asian Pac J Cancer Prev, 16 (18), 8451-8454

\section{Introduction}

Gallbladder cancer (GBC) is one of the most invasive malignancies. The incidence of GBC varies widely among different geographic regions, with South Korea having the highest incidence rate, followed by Japan, among the countries in Far East Asia (Randi et al., 2006). According to the annual reports published by the Korea Central Cancer Registry, National Cancer Center, South Korea, the highest regional age-standardized incidence rate (ASIR) of GBC in females was recorded in Jeju Island among the 16 regions in the country during the past 6 years, from 1999 to 2005 (Bae, 2012).

Before this study, we performed a descriptive epidemiological study of GBC based on the data of patients in our institute and suggested that advanced age, female sex, higher parity, and combined benign gallbladder diseases (polypoid lesions of the gallbladder [PLG] and gallstones) should be considered as candidate risk factors of GBC (Cha et al., 2013). On the basis of this previous study, we hypothesized that region-specific causes affect the incidence of GBC in Jeju island. Then, we performed this case-control study to test the relevance of these risk factors of GBC in the population of Jeju Island.

\section{Materials and Methods}

This is a retrospective case-control study in a single institution. The protocol of this study was approved by the Ethical Community of Institutional Review Boards of Cheju Halla General Hospital, South Korea, and was registered at http://www.clinicaltrials.gov (Identifier No: NCT02326948).

\section{Subjects}

Cases were defined as patients newly diagnosed with GBC at the Digestive Disease Center and Department of Internal Medicine of Cheju Halla General Hospital, Jeju, South Korea, between 2008 and 2013. The patients in the control group were 1:1 matched subjects according 
to age and sex distributions of GBC cases and randomly selected among the health-care check-up examinees who visited the health promotion center in the same institute and periods. In the analyses, 78 of the 124 patients with GBC and 78 matched controls from among 7,088 health checkup examinees were included (Figure 1).

The GBC diagnosis was established based on clinical symptoms, laboratory findings, and imaging studies, including computed tomography, magnetic resonance imaging (MRI), and/or positron emission tomography and confirmed based on pathological results in the patients who underwent surgery or biopsy. In the patients who were not available for assessment of their pathological results, we confirmed their cancer diagnosis by conducting a clinical follow-up. All of their results were reviewed by an expert panel composed of a clinician, surgeon, radiologist, and pathologist.

\section{Data collection}

Information about the demographic features, medical histories, parity, alcohol consumption, smoking, body mass index (BMI), and concurrent gallbladder diseases, including gallstone diseases (GSDs) and PLG, of the case and control groups was obtained from medical records. All of the participants or their family caregivers were interviewed by well-trained research interviewers by using a structured questionnaire, and confirmed the abovementioned information.

For medical histories, we collected information about hypertension, diabetes, and vascular occlusive diseases (VODs). Female patients were asked about their total number of pregnancies, including abortion. We asked all of the participants about their history of alcohol consumption and smoking behaviors, which were not indicated in their medical records. To standardize the amount of alcohol consumption, reported alcohol consumption was converted into standard drink, which contains 14 $\mathrm{g}$ of alcohol in various kinds of beverages. They were divided into two categories, namely non-drinker (none or fewer than 3 standard drinks per week [SDW]) and drinker (3 or more SDW). BMI was categorized into two groups, namely $<23$ and $\geq 23 \mathrm{~kg} / \mathrm{m} 2$, which was presented as the Asia Pacific overweight criteria (WHO Expert Consultation Lancet, 2004). Smoking history was categorized into two groups, namely ever-smokers and never-smokers.

To evaluate the concurrent gallbladder-related diseases, all the medical histories, surgical specimens, pathological reports, and radiological findings, including ultrasonography, contrast-enhanced computed tomography, or MRI, of all of the subjects in both groups were reviewed. PLG was defined as an echogenic immobile protrusion from the gallbladder wall into the lumen on ultrasonography. Concurrent biliary stone disease was defined according to the presence of strong intraluminal echogenic materials that were mobile, gravity dependent, or acoustic shadow in the gallbladder, bile ducts, or both.

\section{Statistical analyses}

In the statistical analyses, we converted continuous or ordinary variables into dichotomous variables. The distributions of all the dichotomous variables were presented as frequencies and percentages of total pairs. To compare the statistical differences in the proportion of each dichotomized variables of the matched groups in the univariate analysis, we used the McNemar test (McNemar, 1947).

To estimate the potential risk factors associated with GBC, all the statistically significant variables in the univariate analysis and other important confounding factors were assessed by calculating odds ratios (ORs) and $95 \%$ confidence intervals (CIs) in the multivariate analysis by using conditional logistic regression model. For two-sided tests, a $\mathrm{p}$ value $<0.05$ was considered statistically significant. All statistical analyses were performed with $\mathrm{R}$ version 3.0.2 (The R Foundations of Statistical Computing, Seoul, South Korea).

\section{Results}

Table 1 presents the analysis results and characteristics of the 78 matched case-control pairs. In the histories, including hypertension, diabetes, and VODs, no significant differences were found between the two groups. For alcohol consumption, the proportion of subjects who drank more than $3 \mathrm{SDW}$ was higher in the control group than in the case group. Twenty-nine pairs $(36.7 \%)$ consisted of control drinkers and case non-drinkers, but only 5 pairs $(6.3 \%)$ consisted of control non-drinkers and case drinkers. Sixteen pairs $(20.2 \%)$ consisted of cases with PLG and controls without PLG, and 1 pair (1.3\%)

Table 1. Distribution of Clinical Risk Factors of Gallbladder Cancer in the Case-control Pairs (total $\mathrm{n}=156,78$ matched pairs)

\begin{tabular}{|c|c|c|c|c|c|}
\hline \multirow[t]{2}{*}{ Variables } & \multirow[t]{2}{*}{ Controls } & \multicolumn{2}{|c|}{ Cases } & \multirow{2}{*}{$\begin{array}{c}\text { Total pairs } \\
\text { n }(\%)\end{array}$} & \multirow[t]{2}{*}{ p-value 1} \\
\hline & & $\begin{array}{c}\text { No } \\
\text { n (\%) }\end{array}$ & $\begin{array}{c}\text { Yes } \\
\mathrm{n}(\%)\end{array}$ & & \\
\hline \multirow[t]{2}{*}{ Hypertension } & n No & $34(43.6)$ & $14(17.9)$ & $48(61.5)$ & $\mathrm{NS}$ \\
\hline & Yes & $15(19.2)$ & $15(14.9)$ & $30(29.7)$ & \\
\hline \multirow[t]{2}{*}{ DM } & No & $61(77.2)$ & $12(15.2)$ & $73(92.4)$ & 0.08 \\
\hline & Yes & $4(5.1)$ & $1(1.3)$ & $5(6.3)$ & \\
\hline \multirow[t]{2}{*}{ VOD } & No & $65(83.3)$ & $6(7.7)$ & $71(91.0)$ & NS \\
\hline & Yes & $7(9.0)$ & $0(0.0)$ & $7(9.0)$ & \\
\hline \multirow[t]{2}{*}{ Alcohol $^{2}$} & $<3 \mathrm{SDW}$ & $39(49.4)$ & $5(6.3)$ & $44(55.7)$ & $<0.001$ \\
\hline & $\geq 3 \mathrm{SDW}$ & $29(36.7)$ & $5(6.3)$ & $24(43.0)$ & \\
\hline \multirow[t]{2}{*}{ Smoking } & No & $59(77.6)$ & $4(5.3)$ & $63(82.9)$ & 0.121 \\
\hline & Yes & $11(14.5)$ & $2(2.6)$ & $13(17.1)$ & \\
\hline \multirow[t]{2}{*}{ Pregnancy } & $\leq 1$ & $0(0.0)$ & $12(15.2)$ & $12(15.2)$ & 0.016 \\
\hline & $\geq 2$ & $2(2.5)$ & $32(40.5)$ & $34(43.0)$ & \\
\hline \multirow[t]{2}{*}{ Overweight $^{3}$} & No & $21(26.9)$ & $18(46.2)$ & $39(50.0)$ & 0.863 \\
\hline & Yes & $16(41.0)$ & $23(29.5)$ & $39(50.0)$ & \\
\hline \multirow[t]{2}{*}{ PLG } & No & $60(75.9)$ & $16(20.2)$ & $76(96.2)$ & $<0.001$ \\
\hline & Yes & $1(1.3)$ & $1(1.3)$ & $2(2.5)$ & \\
\hline \multirow[t]{2}{*}{ GBS } & No & $61(77.2)$ & $15(19.1)$ & $76(96.2)$ & $<0.001$ \\
\hline & Yes & $2(2.5)$ & $0(0.0)$ & $2(2.5)$ & \\
\hline \multirow[t]{2}{*}{ GSD } & No & $54(68.4)$ & $22(27.9)$ & $76(96.2)$ & $<0.001$ \\
\hline & Yes & $2(2.5)$ & $0(0.0)$ & $2(2.5)$ & \\
\hline
\end{tabular}

${ }^{1}$ Estimated by using the McNemar test; ${ }^{2}$ Alcohol consumption was classified according to standard drinks $($ alcohol amount $=14 \mathrm{~g}$ ) per week; ${ }^{3}$ Defined as a body mass index $\geq 23 \mathrm{~kg} / \mathrm{m}^{2}$; Abbreviations: NS, not significant; DM, diabetes melitus; VOD, vascular occlusive disease; SDW, standard drinks per week; PLG, polypoid lesions of gallbladder; GSD, gallstone disease 
Table 2. The Matched Odds Ratios (mOR) of the Multiple Predicted values Correlating with GB Cancer in the Cases Matched for Age and Sex by using Conditional Cogistic Regression Model. $(n=78$ matched pairs)

\begin{tabular}{llll}
\hline Variables & $\mathrm{p}$ Value & $\operatorname{Exp}(\mathrm{B})$ & $95 \% \mathrm{CI}$ \\
\hline Hypertension & 0.066 & 0.29 & $0.08-1.09$ \\
DM & 0.033 & 2.49 & $0.40-15.7$ \\
VOD & 0.775 & 1.29 & $0.22-7.62$ \\
1Alcohol & $0.00140 * *$ & 0.15 & $0.05-0.48$ \\
Smoking & 0.119 & 0.22 & $0.03-1.48$ \\
2Overweight & 0.55 & 0.74 & $0.28-1.97$ \\
PLG & $0.00927 * *$ & 37.1 & $2.44-564.40$ \\
GSD & $0.00607 * *$ & 21.79 & $2.41-196.9$ \\
\hline
\end{tabular}

**, $\mathrm{p}<0.001 ; 1$ Alcohol consumption was classified according to standard drinks per week (SDW, $<3 v s \geq 3$ ); 2 Defined as body mass index $>=23 \mathrm{~kg} / \mathrm{m}^{2}$; Abbreviations: DM, diabetes mellitus; VOD, vascular occlusive disease; BMI, body mass index; PLG, polypoid lesions of gallbladder; GSD, gallstone disease

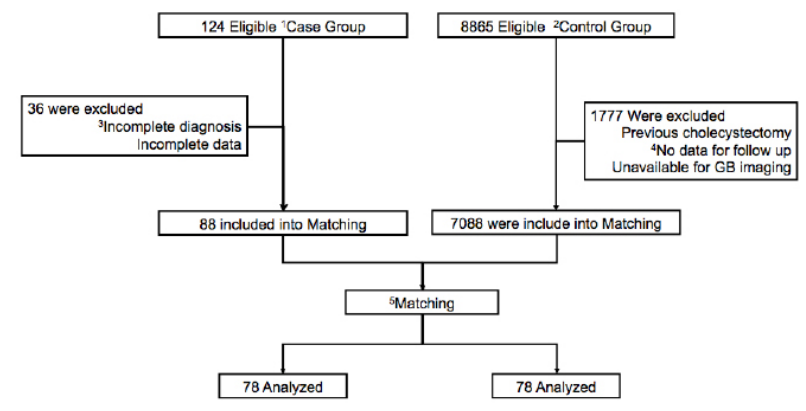

Figure 1. Schematic Flow Diagram of Enrollment and Matching Process of Case and Control gGroup. (Total 78 pairs analyzed)

consisted of a case without PLG and a control with PLG. Significant differences in GSD between the matched groups were also observed.

In the multivariate analysis using a conditional logistic regression model, PLG and GSD were revealed to have strong positive predictive values for $\mathrm{GBC}$ occurrence (PLG: OR, 37.1; 95\% CI, 2.44-564.4; $\mathrm{p}=0.009$; GSD: OR, 21.8; 95\% CI, 2.41-196.9; $\mathrm{p}=0.006$ ). (Table 2). Alcohol consumption of more than 3 SDW had a negative correlation with GBC risk (OR, 0.15; 95\% CI, 0.05-0.48; $\mathrm{p}=0.001)$.

\section{Discussion}

Based on the results of this analytic epidemiological study, we identified the region-specific risk factors of the relatively higher incidence of GBC in this age- and sexmatched case-control study, and found that PLG and GSD had a significant correlation with GBC risk. We observed a negative relationship between the amount of alcohol ingestion and the risk of GBC.

In our study, we failed to prove the relationship between overweight (BMI $\geq 23 \mathrm{~kg} / \mathrm{m} 2$ ) and GBC risk. We also performed a subgroup analysis by using different BMI criteria such as obesity (BMI $\geq 30 \mathrm{~kg} / \mathrm{m} 2$ ) and compared numerical variables regarding BMI. However, we observed the same negative results. Many researchers insisted that higher BMI is correlated with higher GBC risk (Strom, 1995; Zatonsik, 1997; Oh, 2002; Calle, 2003; Ahrens, 2007). This discrepancy could be caused by the time of BMI measurement. Because the case group consisted of many patients with advanced GBC who lost weight due to disease progression at the time of diagnosis, their BMI might have been underestimated.

We also achieved the same correlation between benign gallbladder diseases such as PLG and gallstones (PLG: OR, 37.1; GSD: OR, 27.9). These results showed much stronger predictive values for GBC than other previous documented studies in other regions (Randi, 2006).

Some studies reported alcohol consumption as an important risk factor of GBC (Ozasa, 2007; Yagyu, 2008), and others suggested no relationship between alcohol consumption and GBC (Chow, 1994). Furthermore, several documents report a negative relationship between the two (Ying, 2011). In this study, we observed a negative relationship between alcohol consumption and GBC occurrence. This result is inconsistent to a recent published meta-analysis regarding the various cancer risks from alcohol consumption and suggested that it as a positive predictive factor of GBC (Bagnardi et al., 2015). However, it is interesting that consumptions. And the authors suggested that drinking is a positive this comprehensive dose-response meta-analysis showed that the difference in hazard ratio was dependent on the amount of alcohol consumptions as follows: light $(<12.5$ g/day), 1.23 ; moderate ( $<50 \mathrm{~g} /$ day $), 0.88$; and heavy $(>50$ $\mathrm{g} /$ day), 2.64. Unlike previous studies, our study revealed that an alcohol consumption $>3 \mathrm{SDW}$ ( $6 \mathrm{~g} /$ day) negatively correlated with GBC risk. In addition, we performed the same multivariate analysis but with different categories of amount alcohol consumption as follows: never-drinker or drinker, $\leq 7 \mathrm{SDW}$ (14 g/day), and $\leq 14 \mathrm{SDW}$ (28 g/day). We obtained different results. These features may be explained by the possibility that mild to moderate drinking of alcohol is negatively correlated with GBC occurrence, consistent with the estimated risk of subgroups within the previous meta-analytic study. However, these risks, as reported by Bagnari et al. (2015), may need modification for two reasons. They estimated relative risk from 10 previous published studies, of which some included other bile duct cancers with GBCs. They included only one study that showed a negative correlation between alcohol consumption and GBC. The results suggest that a certain amount of alcohol consumption may have a preventive effect on GBC and is not a risk factor. Regardless of amount of alcohol comsumption, alcohol may have a role as a modifier or by-stander between benign gallbladder disesases (PLG and GSD) and GBC. But we found that alcohol consumption was an independent predictive factor of GBC after adjusting for the effects of PLG and GSD in the multivariate analysis. One study suggested that alcohol consumption correlated negatively with benign gallbladder diseases (Liu, 2009). However, more research studies are required to prove this preventive effect according to the specific amount of alcohol intake.

This study has two limitations, which are regarding its sample size and representativeness. We enrolled 78 subjects each for the case and control groups, but we tried to overcome this limitation of a small sample size 


\section{Byung Hyo Cha}

by conducting a case-control study, matching the ages and sexes of the subjects in both groups. The single-institute survey conducted in this study can raise the argument of whether the GBC control group is representative of the general population of Jeju Island. However, there was only one expert on GBC and the institute was biggest tertiary referral center in the area during the study period. The interregional population drift is relatively lower than that in other provinces in South Korea due to the geographic characteristics of the island.

In conclusion, we found that PLG and GSD are potential risk factors of GBC occurrence in the population of Jeju Island in South Korea in this clinical based ageand sex-matched case-control study. We also observed the negative correlation between mild to moderate alcohol consumption and GBC in this area. Based on our findings, we suggest a detailed survey of these predisposing factors of GBC and early intervention for patients in order to reduce the incidence of this highly aggressive malignant disease. Regarding alcohol drinking, further well-designed cohort study or meta-analysis should be conducted, including many GBC-specific epidemiological studies.

\section{Acknowledgements}

I deeply appreciate Prof. Jong-Myun Bae, M.D. in Jeju National University School of Medicine for great help regarding the study design and statistical analysis.

\section{References}

Ahrens W, Timmer A, Vyberg M, et al (2007). Risk factors for extrahepatic biliary tract carcinoma in men: medical conditions and lifestyle: results from a European multicentre case-control study. Eur J Gastroenterol Hepatol, 19, 623-30.

Alvi AR, Siddiqui NA, Zafar H (2011). Risk factors of gallbladder cancer in Karachi-a case-control study. World J Surg Oncol, 9, 164.

Bae J-M (2012). Regional difference in the occurrence of cancers on biliary system in Korea: a descriptive epidemiological study. Korean Public Health Res, 38, 49-55.

Bagnardi V, Rota M, Botteri E, et al (2015). Alcohol consumption and site-specific cancer risk: a comprehensive dose-response meta-analysis. Br J Cancer, 112, 580-93.

Calle, EE, Rodriguez C, Walker-Thurmond K (2003). Overweight, obesity, and mortality from cancer in a prospectively studied cohort of U.S. adults. New Engl J Med, 348, 1625-38.

Cha BH, Bae JM (2014). Comparison of clinical outcomes of incidental gallbladder cancers: a single-center crosssectional study. Asian Pac J Cancer Prev, 15, 1281-3.

Chow WH, McLaughlin JK, Menck HR, et al (1994). Risk factors for extrahepatic bile duct cancers: Los Angeles County, California (USA). Cancer Causes Control, 5, 267-72.

Li Y, Yang H, Cao J (2011). Association between alcohol consumption and cancers in the Chinese population: a systematic review and meta-analysis. PLoS One, 6, 18776.

Liu B, Balkwill A, Roddam A, et al (2009). Separate and joint effects of alcohol and smoking on the risks of cirrhosis and gallbladder disease in middle-aged women. Am J Epidemiol, 169, 153-60.

McNemar Q (1947). Note on the sampling error of the difference between correlated proportions or percentages. Psychometrika, 12, 153-7.
Ozasa, K (2007). Alcohol use and mortality in the Japan Collaborative Cohort Study for Evaluation of Cancer (JACC). Asian Pac J Cancer Prev, 8, 81-8.

Randi G, Franceschi S, La Vecchia C (2006). Gallbladder cancer worldwide: geographical distribution and risk factors. Int $J$ Cancer, 118, 1591-602.

Strom BL, Soloway RD, Rios-Dalenz JL, et al (1995). Risk factors for gallbladder cancer: an international collaborative case-control study. Cancer, 76, 1747-56.

WHO Expert Consultation (2004). Appropriate body-mass index for Asian populations and its implications for policy and intervention strategies. Lancet, 363, 157-63.

Yagyu K, Kikuchi S, Obata Y (2008). Cigarette smoking, alcohol drinking and the risk of gallbladder cancer death: a prospective cohort study in Japan. Int J Cancer, 122, 924-9.

Zatonski WA, Lowenfels AB, Boyle P, et al (1997). Epidemiologic aspects of gallbladder cancer: a case-control study of the SEARCH Program of the International Agency for Research on Cancer. J Natl Cancer Inst, 89, 1132-8. 